\title{
Diurnal variation in salivary cortisol across age classes in Ache Amerindian males of Paraguay
}

\section{Citation}

Amir, Dorsa, Peter T. Ellison, Kim R. Hill, and Richard G. Bribiescas. 2014. "Diurnal Variation in Salivary Cortisol Across Age Classes in Ache Amerindian Males of Paraguay." Am. J. Hum. Biol. 27 (3) (October 18): 344-348. doi:10.1002/ajhb.22645.

\section{Published Version}

doi:10.1002/ajhb.22645

\section{Permanent link}

http://nrs.harvard.edu/urn-3:HUL.InstRepos:25201565

\section{Terms of Use}

This article was downloaded from Harvard University's DASH repository, and is made available under the terms and conditions applicable to Open Access Policy Articles, as set forth at http:// nrs.harvard.edu/urn-3:HUL.InstRepos:dash.current.terms-of-use\#OAP

\section{Share Your Story}

The Harvard community has made this article openly available.

Please share how this access benefits you. Submit a story.

\section{Accessibility}


Diurnal variation in salivary cortisol across age classes in Ache Amerindian males of Paraguay

${ }^{1 *}$ Amir, D., ${ }^{3}$ Ellison, P.T., ${ }^{2}$ Hill, K.R., and ${ }^{1}$ Bribiescas, R.G.,

1Yale University, Reproductive Ecology Laboratory, Department of Anthropology, 10

Sachem Street, New Haven, CT 06520-8277.

${ }^{2}$ Arizona State University, School of Human Evolution and Social Change, Tempe, AZ.

${ }^{3}$ Harvard University, Department of Human Evolutionary Biology, 11 Divinity Avenue, Cambridge, MA 02138

* Corresponding author, email address: dorsa.amir@yale.edu 


\section{Abstract:}

Objectives: Cortisol levels exhibit a diurnal rhythm in healthy men, with peaks in the morning and troughs in the evening. Throughout age, however, this rhythm tends to flatten. This diurnal flattening has been demonstrated in a majority of industrialized populations, though the results have not been unanimous. Regardless, little attention has been paid to non-industrialized, foraging populations such as the Ache Amerindians of Paraguay. As testosterone levels had previously been shown to diminish with age in this population (Bribiescas and Hill, 2010), we hypothesized that cortisol levels would behave similarly, flattening in rhythmicity over age.

Methods: We examined morning and evening salivary cortisol samples in Ache Amerindian men in association with age $(n=40$, age range: $20-64$ years).

Results: Men in the first age class (<20-29 years) exhibited significantly different $\mathrm{AM}$ and PM values as did men in the second age class (30-39 years). However, men in the third and fourth age classes (40-49 years, and $>50$ years, respectively) did not exhibit a significant difference between AM and PM values.

Conclusion: Ache Amerindian men exhibit a flattening of the diurnal rhythm across age classes. Our results were able to capture both within- and between-individual variations in cortisol levels, and reflected age-related contrasts in daily cortisol fluctuations. The flattening of the diurnal rhythm with age among the Ache may reflect a common and shared aspect of male senescence across ecological contexts and lifestyles.

\section{Introduction:}

Cortisol is the main glucocorticoid hormone in humans and most other vertebrates. It is produced in the adrenal cortex both spontaneously and as a response to biochemical agents and psychosocial stimuli (Kirschbaum and Hellhammer, 1989). Cortisol is regulated by the hypothalamic-pituitary-adrenal axis (HPA), which is involved in homeostatic and allostatic adjustments to both internal and external stressors (Chahal and Drake, 2007). During a stress response, increases in corticotropic releasing hormone (CRH) by the paraventricular nucleus in the hypothalamus stimulates production of adrenocorticotropic hormone (ACTH) by the pituitary gland, leading to increased production of of cortisol by the cortical region of the adrenal gland. Cortisol assists in glycogenolysis, the breakdown of glycogen to release glucose, lipolysis, the release of fatty acids from fat stores, suppression of the immune system, and the distribution of fat in the body. As it increases in response to stressors, cortisol has gained the moniker of 'stress hormone', though its primary functions are metabolic. Along with other endocrine hormones such as testosterone (Cooke et al., 1993), cortisol exhibits a well-documented circadian pattern in healthy human males, in which morning levels are typically higher than evening levels (Clow et al., 2010; Faiman and Winter, 1971; Hayes et al., 2012; Hucklebridge et al., 2005; Van Cauter et al., 1996).

Age appears to be an important factor in adrenal function and subsequent levels of available cortisol. Studies have shown that overall average levels of cortisol decrease with age (Drafta et al., 1982; Sherman et al., 1985), but that there are significant age-associated increases in minimal cortisol concentrations (Deuschle et al., 1997). Studies assessing HPA 
axis responses to cognitive challenges also report a significant increase in baseline levels of cortisol with age (Seeman et al., 2001). In addition, older adults have been shown to exhibit a blunted cortisol awakening response when compared to younger adults (Heaney et al., 2010). Importantly, older adults exhibit significantly reduced diurnal slopes and a flatter diurnal rhythm (Deuschle et al., 1997; Heaney et al., 2010; Van Cauter et al., 1996).

Changes in the diurnal rhythm of cortisol across age are most likely due to changes in the functioning of the HPA axis. In rodents, there is an age-related reduction in the sensitivity of the HPA axis to glucocorticoid feedback inhibition (Sapolsky et al., 1986a), along with an increase in HPA axis responsiveness in older rodents (Sapolsky et al., 1986b). This pattern is also observed in humans. In aging individuals, endocrine changes result in a decline in endocrine function associated with the responsiveness of tissues and reduced hormone secretion from peripheral glands (Chahal and Drake, 2007). In the HPA axis, specifically, the main alteration throughout age appears to be diminished sensitivity to glucocorticoid feedback inhibition in older adults (Chahal and Drake, 2007), as there appears to be no deficiency of adrenal corticosteroid production associated with aging (Waltman et al., 1991). During aging, there also appears to be a progressive decline in the endogenous inhibition of nocturnal cortisol secretion (Van Cauter et al., 1996).

Age-related changes in HPA axis function may have significant effects on both psychology and physiology. An increase in the levels of HPA axis activity and increased levels of circulating cortisol have been linked to the development of cognitive impairment (Karlamangla et al., 2005; MacLullich et al., 2005). Lupien and colleagues demonstrated that recent evidence of HPA dysregulation is associated with impaired cognitive performance in a battery of memory, attention and language tests (Lupien et al., 1994), later suggesting that cumulative exposure to high levels of glucocorticoids such as cortisol are detrimental to the aged hippocampus and may be contributing to diseases such as dementia and depression (Lupien et al., 1999). Cortisol levels have also been shown to be inversely associated to bone mineral density and rate of bone loss in elderly men, suggesting that the endogenous cortisol profiles is a determinant of bone health (Dennison et al., 1999). Additionally, cortisol has been implicated as a contributing factor for the agerelated decline in immune function termed immunosenescence (Buford and Willoughby, 2008).

As most scientific studies have been conducted in Western, Educated, Industrialized, Rich, and Democratic (WEIRD) populations (Henrich et al., 2010), it is not surprising that there is a paucity of studies examining HPA axis function in non-WEIRD populations. This data is important in establishing whether certain patterns in senescence are conserved across human populations or whether there are changes in these patterns with changing environment. If there are differences between populations in HPA function, we can then answer the question of why they exist. Several studies have shed light on human cortisol function across the world, with data from the Caribbean (Decker, 2000; Flinn and England, 1997), Mexico (Fernald et al., 2009), Kenya (Pike and Williams, 2006), Guatemala (Nepomnaschy et al., 2004), Mongolia (Hruschka et al., 2005), Kenya (Gray, 2003) and Nepal (Hruschka et al., 2005; Worthman and Panter-Brick, 2008). However, few studies have been conducted among foraging populations, which are important when studying human evolutionary biology as these populations live in environments more similar to those of their ancestors when compared to WEIRD populations. Only one study to date (Nyberg, 2012) has measured diurnal cortisol rhythmicity in an indigenous foraging 
population.. That study found dramatically lower levels of HPA activity among the Tsimané of Amazonian Bolivia, with a flattened diurnal cortisol pattern in both men and women over the age of 60, when compared with Western data from the CARDIA study (Nyberg, 2012).. As such, we predicted that cortisol would behave similarly, exhibiting a flattened diurnal pattern with increasing age.

The participants in this study were 40 healthy Ache men from the community of Chupa Pou in eastern Paraguay. At the time of this investigation, this community subsisted on a mix of foraging for wild game and plant products from the surrounding forest, as well as on manual agriculture. For more details on Ache ecology and culture, see Hill and Hurtado (1996). This investigation protocol and recruitment were approved by the Harvard University Human Subjects Committee.

\section{Methods:}

Data for this investigation was obtained from archived salivary cortisol values obtained during an investigation of male reproductive endocrinology in 1992. Salivary cortisol was assayed in 1993 using methods outlined below. Healthy Ache men ( $\mathrm{n}=40$, age range: 20-64 years) were recruited for this study. Subjects were screened for acute and chronic health conditions such as upper respiratory disease and tuberculosis and reported no developmental abnormalities or metabolic conditions that may affect cortisol levels (ie., Addison's or Cushing's Disease). Morning ( $\sim 6 \mathrm{am})$ and evening ( $\sim 6 \mathrm{pm})$ salivary samples (approximately $2 \mathrm{mLs}$ ) were collected from each subject for $1-10$ days (mean samples/individual AM N = $5.2 \pm 0.3 \mathrm{SE} ; \mathrm{PM} \mathrm{N}=5.1 \pm 0.3$ ). Saliva was collected using the methods of Lipson and Ellison (1989) in $12 \times 75 \mathrm{~mm}$ polystyrene tubes pretreated with sodium azide as a preservative. Samples were stored at ambient temperature for 3 weeks before shipment to the Reproductive Ecology Laboratory at Harvard University. Upon arrival, samples were stored frozen at $-20^{\circ} \mathrm{C}$ to facilitate the breakdown of muccopolysaccharides until assayed.

Salivary cortisol was measured by direct radioimmunoassay using $50 \mu \mathrm{l}$ of saliva, a four position tritiated competitor (Amersham, Arlington Heights IL), and a steroid specific antibody. Samples were incubated at $4^{\circ} \mathrm{C}$ for 24 hours with the unbound fraction separated using a dextran coated charcoal suspension. The bound fraction was then counted in a liquid scintillation counter and analyzed using the log/logit method. Inter-assay variability was calculated using coefficients of variation (cv) in high, medium, and low quality controls (high $10.9 \mathrm{nmol} / \mathrm{L} \pm 0.24 \mathrm{se}, \mathrm{cv}=8.0 \%$, medium $4.0 \pm 0.13, \mathrm{cv}=11.3 \%$, low $2.3 \pm 0.07, \mathrm{cv}=$ $11.0 \%$ ). Quality control blanks consistently read below detection ranges.

Anthropometric measurements were determined as follows. Height was determined using a standard anthropometer on a solid surface with the head in the Frankfurt plane, weight using a standard scale to the nearest $0.1 \mathrm{~kg}$, and BMI (body mass index) calculated using weight $/$ height $^{\wedge} 2\left(\mathrm{~cm} / \mathrm{kg}^{\wedge} 2\right)$.

The Ache do not keep written records of birth dates, although some births were recorded by missionaries and through informants. Age was ascertained from known birth dates, and cross-referencing with known events. The specific methods of aging Ache individuals have been described elsewhere (Hill and Hurtado, 1996, Chapter 4). 
Means, standard errors, and standard deviations were calculated for both AM and PM cortisol levels. Comparisons of average cortisol levels and age classes were conducted using repeated measures ANOVA(means $\pm \mathrm{SD}$ ). Statistical analysis was conducted using STATA 13 (StataCorp, College Station, TX). Alpha was set at 0.05 .

Results:

A summary of the data collected is presented in Table 1, categorized by age class $(\mathrm{N}=40$, average age $=36.5 \pm 1.8$ years, age range $=20$ - 64 years) .

\section{Cortisol vs. Age Class}

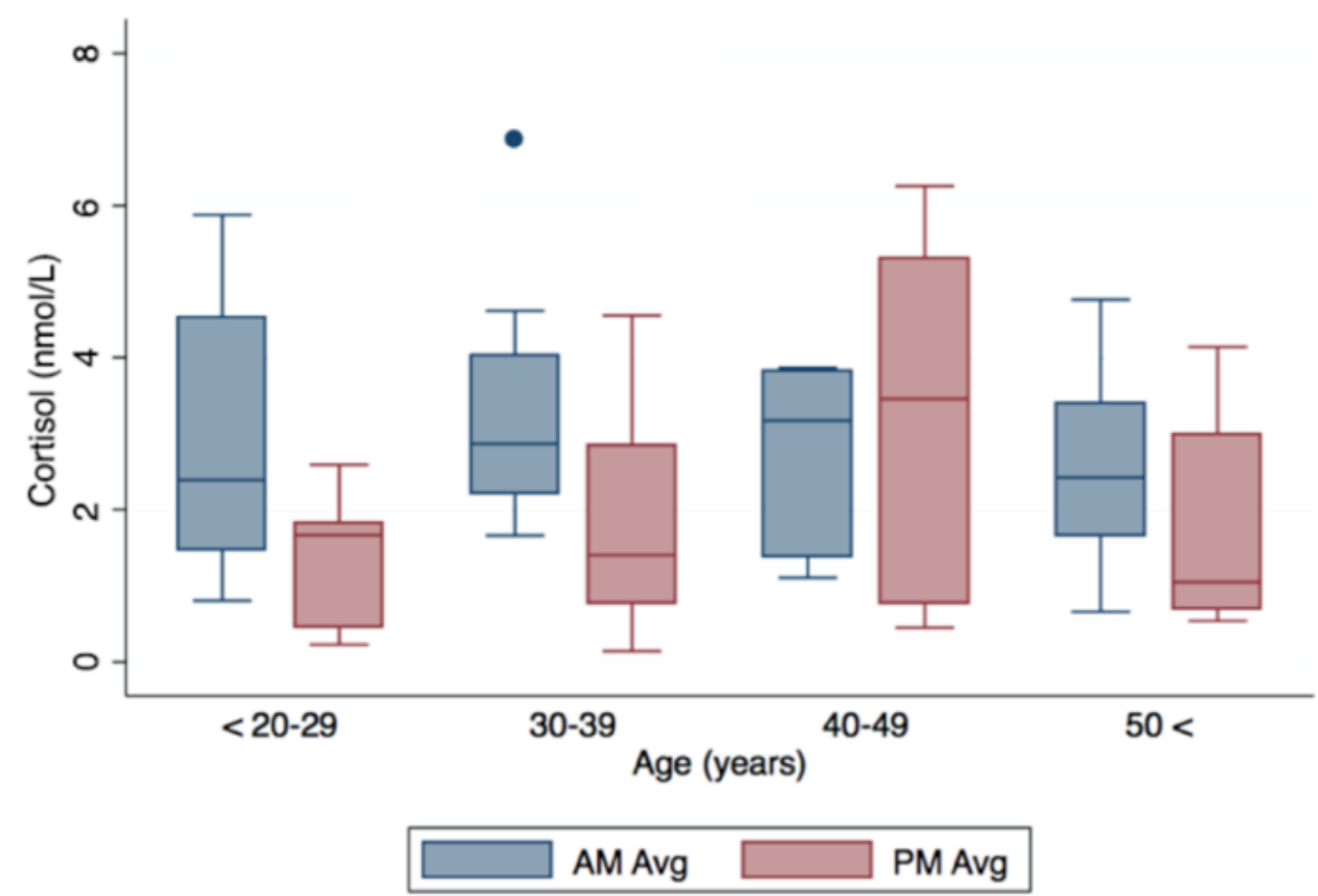

Fig. 1: Average AM cortisol values and average PM cortisol values for Ache men, categorized by age class.

We assessed the difference between average AM cortisol values and average PM cortisol values within each age class, presented in Figure 1. All analysis was done against the null hypothesis that the AM and PM values of cortisol are equal within any given age class. We first binned participants into four age classes, based on age at time of collection. Decade-based age classes were used to be consistent with previous investigations that examined diurnal hormone biology and age in men (Bribiescas and Hill, 2010; Uchida et al., 2006). As previously demonstrated, the use of decades is a convenient way to categorize 
males who have completed the pubertal transition (those older than 20). Other studies used age groups that spanned 15 years (Ellison et al., 2002); however, this investigation had unique sample size constraints that involved the use of meta-data from several studies.

We ran a repeated measures ANOVA to determine the effect of time of day (AM or PM), age class, and the time of day by age class interaction on cortisol levels. We found a main effect of time of day such that samples taken in the morning $(M=2885.29$, $\mathrm{SD}=1384.87)$ have a larger mean than samples taken in the evening $(\mathrm{M}=1877.11$, $\mathrm{SD}=1537.62), \mathrm{F}(1,39)=8.36, \mathrm{p}=0.006$. We found no main effect of age class on cortisol level, $F(3,39)=1.10, p=0.361$. However, we found a marginally significant time of day by age class interaction such that as age class increases, the difference between AM and PM cortisol levels decreases $F(3,39)=2.59$, $p=.062$.

Given the precedent in the literature for effects of age on cortisol levels, we wanted to ensure that the main effect we failed to find of age on cortisol levels in the ANOVA was valid. Using age as a continuous variable in univariate regressions, we were unable to predict AM cortisol levels $(\beta=-9.15, S E=19.73, p=0.646)$, PM cortisol levels $(\beta=28.14$, $\mathrm{SE}=21.49, \mathrm{p}=0.198)$, or the AM:PM ratio $(\beta=-0.02, \mathrm{SE}=0.04, \mathrm{p}=0.570)$.

\section{Discussion:}

In this analysis, we found that Ache Amerindian men exhibit a flattening of the diurnal rhythm across age classes. This study is the first to document changes in cortisol levels across age in a small-scale foraging society from Amazonian Paraguay. While population comparisons may provide insights into male senescence across cultures, between- and within-individual variation is necessary to capture central sources of hormone variation (Bribiescas, 2005). Our methods allowed us to analyze variations both within individuals and between individuals, in order to produce a more complete and finegrained understanding of how reproductive senescence occurs in males.

Our work aligns with the majority of previous work showing a flattening of the diurnal slope of cortisol with age among men. Table 2 provides a comparison of studies analyzing age-related changes in cortisol across different populations. While the pattern of slope flattening does not appear to be fully consistent across populations, there may be several variables affecting the power of these comparisons. First, not all studies are extracting cortisol levels from the same fluid; half of the studies sampled blood, while the other half sampled saliva. Salivary cortisol has been shown to be an effective measure of levels of free, circulating cortisol, but while the correlation between plasma-derived and salivary cortisol is highly significant, it is not always an exact match (Kahn et al., 1988). Second, assays were performed in different laboratories, increasing the possibility of interlaboratory variation. Third, the age ranges of the studies are not consistent, with a major difference being that studies exhibiting diurnal flattening all included individuals above the age of 63. The one exception to this pattern is our study, in which the maximum age sampled was 64. While it is possible that Ache men exhibit a more rapid flattening of cortisol rhythm with age, this result may be influenced by sample size, as samples were twice as likely to be taken from men in the first two age classes than from the latter two. 
An important finding is that the overall cortisol levels of Ache men are significantly lower than their Western counterparts. Figure 2 depicts average AM and PM values between Ache men in the first age class ( $<20-29$ years) to similarly aged American individuals (18-30 years). The American data was extracted from the CARDIA study (Cohen et al., 2006). While this particular CARDIA data aggregates both male and female samples, gender variations in cortisol levels are not significantly different enough to obscure the observation that Ache men have extremely low cortisol levels when compared to American counterparts.

\section{Ache vs. Americans, Age Class 1}

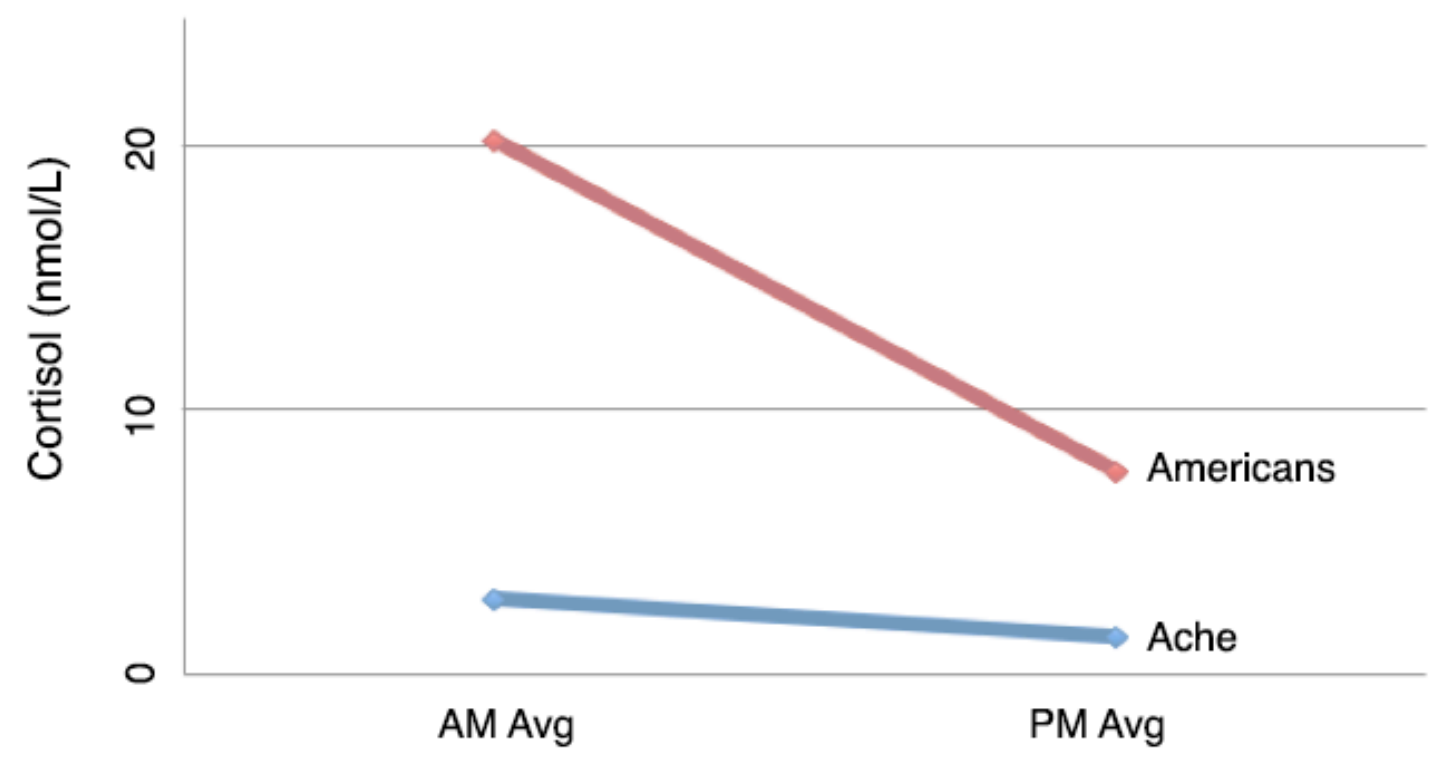

Fig. 2: Average AM and PM cortisol values for the first age class ( $<20-29$ years) among Ache men and American individuals. American data is taken from the CARDIA study (Cohen et al., 2006).

What accounts for the extreme difference between American and Ache cortisol levels? First, it is important to note that the endocrinological differences between the Ache and their Western counterparts are not limited to cortisol; similar trends have been demonstrated in both testosterone (Bribiescas, 1996) and leptin (Bribiescas, 2005). In fact, leptin levels in Ache women were so low that they were comparable to anorectic American women, despite major differences in fat percentage (Bribiescas, 2005). Some of the variation in cortisol levels may be explained through differences in energetics. Among the Ache, there is little seasonality in nutrient availability and there appears to be no cyclical change in physical work (Hill et al., 1984). A recent study has shown that while physical 
activity levels differ among hunter-gatherers and those living in market economies, total energetic expenditure is remarkably similar between these populations (Pontzer et al., 2012). Therefore, it's likely that dietary differences play a more important role in crosscultural variation in hormone levels. An evolved sensitivity of the HPA axis to energy status may be a pathway in which environmental conditions are reflected in cortisol rhythm variations (Nyberg, 2012). Similarly, it has been demonstrated that the sensitivity of hypothalamic-pituitary-gonadal axis (HPG) is shaped by the environment in which it develops (Ellison et al., 1993), and may be acted on epigenetically to convey information cross-generationally (Kuzawa and Quinn, 2009). The discrepancy between the hormones of industrialized and non-industrialized individuals continues to be investigated.

The flattening of the diurnal cortisol rhythm may be attributed to decreased sensitivity in the hypothalamus. While adrenal corticosteroid production is relatively consistent across age (Waltman et al., 1991), there is diminished sensitivity to glucocorticoid feedback inhibition with increasing age (Chahal and Drake, 2007). This means that cortisol is being released at the same rate, but building up over time as agerelated wear and tear diminishes the hypothalamus's sensitivity to negative feedback. This aligns with the finding that alterations of basal cortisol secretion are not restricted to senescence, but begin in the first third of adult life and then gradually become more evident with increasing age (Kern et al., 1996).

During aging, there also appears to be a progressive decline in the endogenous inhibition of nocturnal cortisol secretion (Van Cauter et al., 1996). Amount of and quality of sleep may be important. The mean percentage of deep, slow-wave sleep decreases from $18.9 \%$ during early adulthood (16-25 years) to 3.4\% in midlife (36-50 years), and was replaced by lighter sleep (stages 1 and 2) (Van Cauter et al., 2000). This is paralleled by a major decline in GH secretion across age, and an increase in evening cortisol concentrations (Van Cauter et al., 2000). The increase in nighttime cortisol values across age can help explain the flattening of the diurnal rhythm. Further, analyses suggest that a relationship exists between HPA impairment and decreased amounts of REM sleep, independently of age, suggesting that decreased sleep quality contributes to the allostatic load, or the wear-and-tear, resulting from overactivity of stress-responsive systems (Van Cauter et al., 2000). In the forest, sleep patterns may be altered by amount of available food, group festivity, and men may wake up in the middle of the night to stoke fires or sing (Hill, personal observation). In general, Ache men go to sleep around $9 \mathrm{pm}$ and wake up at sunrise, though men over the age of 50 tend to sleep longer into the day and are more likely to be awake for a period during the night; however, these trends are not strong (Hill, personal observation).

There are several limitations in this study. One is that sample size is not even throughout the age classes, which reduces power in the statistical analyses. However, the quantity of individuals in each age class was more or less representative of the study population. Furthermore, while the majority of men in this study exhibited a classic diurnal pattern of higher AM levels and lower PM levels, 11 subjects did not. These subjects ranged in age from 21 to 64, so age did not appear to be a significant predicting factor. Further, this difference cannot be accounted for by absolute number of samples, as the average number of samples within the classic and non-classic rhythms was not significantly different.

Another confound in this study is the lack of stress measures to evaluate distress or chronic stress, along with lack of sleep/wake data in the analysis. 
In summary, this study reveals age-related changes in cortisol levels among Ache men. Results contribute to a growing body of knowledge regarding alterations of the hypothalamus-pituitary-adrenal axis in aging. Importantly, these results indicate that while differences exist in absolute levels of cortisol across populations, there appear to be some patterns in senescence that are more conserved across different ecological contexts and lifestyles. Further, our results were able to capture important within- and betweenindividual variations, which produced a more fine-grained picture of how cortisol changes across time. Future investigations should attempt to capture this diurnal pattern change in a wider variety of environments and populations, and analyze more quantitatively the contribution of amount and quality of sleep to diurnal pattern changes.

\section{Acknowledgments:}

The authors would like to thank Matthew Jordan for his helpful comments and analysis throughout the preparation of this manuscript.

\section{Literature cited:}

Bribiescas RG. 1996. Testosterone levels among Ache hunter-gatherer men : A functional interpretation of population variation among adult males. Human nature 7(2):163188.

Bribiescas RG. 2005. Serum leptin levels in Ache Amerindian females with normal adiposity are not significantly different from American anorexia nervosa patients. American Journal of Human Biology 17(2):207-210.

Bribiescas RG, Hill KR. 2010. Circadian variation in salivary testosterone across age classes in Ache Amerindian males of Paraguay. Am J Hum Biol 22(2):216-220.

Buford TW, Willoughby DS. 2008. Impact of DHEA (S) and cortisol on immune function in aging: a brief review. Applied Physiology, Nutrition, and Metabolism 33(3):429-433.

Chahal HS, Drake WM. 2007. The endocrine system and ageing. The Journal of pathology 211(2):173-180.

Clow A, Hucklebridge F, Thorn L. 2010. The cortisol awakening response in context. International review of neurobiology 93:153-175.

Cohen S, Schwartz JE, Epel E, Kirschbaum C, Sidney S, Seeman T. 2006. Socioeconomic status, race, and diurnal cortisol decline in the Coronary Artery Risk Development in Young Adults (CARDIA) Study. Psychosomatic medicine 68(1):41-50.

Cooke RR, McIntosh JE, McIntosh RP. 1993. Circadian variation in serum free and nonSHBG-bound testosterone in normal men: measurements, and simulation using a mass action model. Clinical endocrinology 39(2):163-171.

Decker SA. 2000. Salivary cortisol and social status among Dominican men. Hormones and behavior 38(1):29-38.

Dennison E, Hindmarsh P, Fall C, Kellingray S, Barker D, Phillips D, Cooper C. 1999. Profiles of endogenous circulating cortisol and bone mineral density in healthy elderly men. Journal of Clinical Endocrinology \& Metabolism 84(9):3058-3063. 
Deuschle M, Gotthardt U, Schweiger U, Weber B, Körner A, Schmider J, Standhardt H, Lammers C-H, Heuser I. 1997. With aging in humans the activity of the hypothalamus-pituitary-adrenal system increases and its diurnal amplitude flattens. Life sciences 61(22):2239-2246.

Drafta D, Schindler AE, Stroe E, Neacsu E. 1982. Age-related changes of plasma steroids in normal adult males. Journal of steroid biochemistry 17(6):683-687.

Ellison PT, Bribiescas RG, Bentley GR, Campbell BC, Lipson SF, Panter-Brick C, Hill K. 2002. Population variation in age-related decline in male salivary testosterone. Hum Reprod 17(12):3251-3253.

Ellison PT, Panter-Brick C, Lipson SF, O'Rourke MT. 1993. The ecological context of human ovarian function. Human reproduction 8(12):2248-2258.

Faiman C, Winter JS. 1971. Diurnal cycles in plasma FSH, testosterone and cortisol in men. The Journal of clinical endocrinology and metabolism 33(2):186-192.

Fernald LC, Gertler PJ, Neufeld LM. 2009. 10-year effect of Oportunidades, Mexico's conditional cash transfer programme, on child growth, cognition, language, and behaviour: a longitudinal follow-up study. Lancet 374(9706):1997-2005.

Flinn MV, England BG. 1997. Social economics of childhood glucocorticoid stress response and health. American journal of physical anthropology 102(1):33-53.

Gray P. 2003. Human Male Reproductive Strategies: Cross-cultural and Endocrine Aspects: Harvard University.

Hayes LD, Grace FM, KilgoreJl YJD, Baker JS. 2012. Diurnal variation of cortisol, testosterone, and their ratio in apparently healthy males. Sport SPA 9(1):5-13.

Heaney JL, Phillips AC, Carroll D. 2010. Ageing, depression, anxiety, social support and the diurnal rhythm and awakening response of salivary cortisol. International journal of psychophysiology : official journal of the International Organization of Psychophysiology 78(3):201-208.

Henrich J, Heine SJ, Norenzayan A. 2010. The weirdest people in the world? The Behavioral and brain sciences 33(2-3):61-83; discussion 83-135.

Hill K, Hawkes K, Hurtado M, Kaplan H. 1984. Seasonal Variance in the Diet of Ache HunterGatherers in Eastern Paraguay. Hum Ecol 12(2):101-135.

Hill KR, Hurtado AM. 1996. Ache life history: The ecology and demography of a foraging people: Transaction Publishers.

Hruschka DJ, Kohrt BA, Worthman CM. 2005. Estimating between-and within-individual variation in cortisol levels using multilevel models. Psychoneuroendocrinology 30(7):698-714.

Hucklebridge F, Hussain T, Evans P, Clow A. 2005. The diurnal patterns of the adrenal steroids cortisol and dehydroepiandrosterone (DHEA) in relation to awakening. Psychoneuroendocrinology 30(1):51-57.

Kahn J-P, Rubinow DR, Davis CL, Kling M, Post RM. 1988. Salivary cortisol: a practical method for evaluation of adrenal function. Biological psychiatry 23(4):335-349.

Karlamangla AS, Singer BH, Chodosh J, McEwen BS, Seeman TE. 2005. Urinary cortisol excretion as a predictor of incident cognitive impairment. Neurobiol Aging 26 Suppl $1: 80-84$.

Kern W, Dodt C, Born J, Fehm HL. 1996. Changes in cortisol and growth hormone secretion during nocturnal sleep in the course of aging. The journals of gerontology Series A, Biological sciences and medical sciences 51(1):M3-9. 
Kirschbaum C, Hellhammer DH. 1989. Salivary cortisol in psychobiological research: an overview. Neuropsychobiology 22(3):150-169.

Kuzawa CW, Quinn EA. 2009. Developmental origins of adult function and health: evolutionary hypotheses. Annual Review of Anthropology 38:131-147.

Lipson SF, Ellison PT. 1989. Development of the protocols for the application of salivary steroid analyses to field conditions. Am J Hum Biol 1:249-255.

Lupien S, Lecours AR, Lussier I, Schwartz G, Nair NP, Meaney MJ. 1994. Basal cortisol levels and cognitive deficits in human aging. The Journal of Neuroscience 14(5):28932903.

Lupien SJ, Nair NPV, Briere S, Maheu F, Tu MT, Lemay M, McEwen BS, Meaney MJ. 1999. Increased cortisol levels and impaired cognition in human aging: implication for depression and dementia in later life. Reviews in the Neurosciences 10(2):117-140.

MacLullich AM, Deary IJ, Starr JM, Ferguson KJ, Wardlaw JM, Seckl JR. 2005. Plasma cortisol levels, brain volumes and cognition in healthy elderly men.

Psychoneuroendocrinology 30(5):505-515.

Nepomnaschy PA, Welch K, McConnell D, Strassmann BI, England BG. 2004. Stress and female reproductive function: a study of daily variations in cortisol, gonadotrophins, and gonadal steroids in a rural Mayan population. American journal of human biology : the official journal of the Human Biology Council 16(5):523-532.

Nyberg CH. 2012. Diurnal cortisol rhythms in Tsimane' Amazonian foragers: new insights into ecological HPA axis research. Psychoneuroendocrinology 37(2):178-190.

Pike IL, Williams SR. 2006. Incorporating psychosocial health into biocultural models: preliminary findings from Turkana women of Kenya. American journal of human biology : the official journal of the Human Biology Council 18(6):729-740.

Pontzer H, Raichlen DA, Wood BM, Mabulla AZ, Racette SB, Marlowe FW. 2012. Huntergatherer energetics and human obesity. PloS one 7(7):e40503.

Sapolsky RM, Krey LC, McEwen BS. 1986a. The adrenocortical axis in the aged rat: impaired sensitivity to both fast and delayed feedback inhibition. Neurobiology of aging 7(5):331-335.

Sapolsky RM, Krey LC, McEwen BS. 1986b. The neuroendocrinology of stress and aging: the glucocorticoid cascade hypothesis. Endocrine reviews 7(3):284-301.

Seeman TE, Singer B, Wilkinson CW, McEwen B. 2001. Gender differences in age-related changes in HPA axis reactivity. Psychoneuroendocrinology 26(3):225-240.

Sherman B, Wysham C, Pfohl B. 1985. Age-related changes in the circadian rhythm of plasma cortisol in man. The Journal of clinical endocrinology and metabolism 61(3):439-443.

Uchida A, Bribiescas RG, Ellison PT, Kanamori M, Ando J, Hirose N, Ono Y. 2006. Age related variation of salivary testosterone values in healthy Japanese males. Aging Male 9(4):207-213.

Van Cauter E, Leproult R, Kupfer DJ. 1996. Effects of gender and age on the levels and circadian rhythmicity of plasma cortisol. The Journal of clinical endocrinology and metabolism 81(7):2468-2473.

Van Cauter E, Leproult R, Plat L. 2000. Age-related changes in slow wave sleep and REM sleep and relationship with growth hormone and cortisol levels in healthy men. JAMA : the journal of the American Medical Association 284(7):861-868. 
Waltman C, Blackman MR, Chrousos GP, Riemann C, Harman SM. 1991. Spontaneous and glucocorticoid-inhibited adrenocorticotropic hormone and cortisol secretion are similar in healthy young and old men. The Journal of clinical endocrinology and metabolism 73(3):495-502.

Worthman CM, Panter-Brick C. 2008. Homeless street children in Nepal: use of allostatic load to assess the burden of childhood adversity. Development and psychopathology 20(1):233-255. 